\title{
Unitary monodromy of Lamé differential operators
}

\author{
F.Beukers
}

May 17, 2007

\begin{abstract}
The classical second order Lamé equation contains a so-called accessory parameter $B$. In this paper we study for which values of $B$ the Lamé equation has a monodromy group which is conjugate to a subgroup of $S L(2, \mathbb{R}$ ) (unitary monodromy with indefinite hermitian form). We refomulate the problem as a spectral problem and give an asymptotic expansion for the spectrum.
\end{abstract}

\section{Introduction}

Consider the differential equation in the complex plane

$$
P(z) y^{\prime \prime}+\frac{1}{2} P^{\prime}(z) y+\left(\frac{z}{4}-B\right) y=0, \quad P(z)=4 z^{3}-g_{2} z-g_{3}
$$

and where $P(z)$ has three distinct zeros $z_{1}, z_{2}, z_{3}$. This is the Lamé equation with parameter $n=-1 / 2$. See [WW]. This equation is Fuchsian and has four singular points, $z_{1}, z_{2}, z_{3}, \infty$ and local exponents $0,1 / 2$ at the finite singularities and exponents $1 / 4,1 / 4$ at $\infty$. Conversely any linear differential equation with these four singularities and local exponents is necessarily of the form given above. Only the parameter $B$ is not determined by the location of the singularities and their exponents. This parameter is known as the accessory parameter. Let $G$ be the monodromy group of equation (1).

We shall be interested in the following question.

Question 1.1 (Accessory parameter problem) We call $G$ unitary if it admits a nontrivial $G$-invariant hermitian form on $\mathbb{C}^{2}$ (not necessarily positive definite). Given $P(z)$, for which complex values of $B$ is $G$ unitary?

For differential equations in the $p$-adic domain there is the similar question for which values of $B$ there exist solutions with $p$-adic radius of convergence one (see [Dwork]). This problem is studied in a separate paper [Be2002]. We like to consider the present problem as the $\infty$-adic version of it.

There is a very picturesque interpretation of the condition "unitary" on the monodromy group $G$ in the case when $z_{1}, z_{2}, z_{3}$ are real with ordering $z_{1}<z_{2}<z_{3}$. Consider a Schwarz map corresponding to the equation (1) which consists of the quotient $D(z)=y_{1}(z) / y_{2}(z)$ of two independent solutions $y_{1}$ and $y_{2}$ of (1). On the upper half plane this function can be chosen as a one-valued analytic function. Since (1) has real coefficients on $\mathbb{R}$, the Schwarz map maps 
the four segments $\left(-\infty, z_{1}\right),\left(z_{1}, z_{2}\right),\left(z_{2}, z_{3}\right),\left(z_{3}, \infty\right)$ onto four segments of circles, which we denote by $I, I I, I I I, I V$. Moreover, the segments $I$ and $I V$ are tangent, due to the exponent differences zero at $\infty$. The pairs $(I, I I),(I I, I I I),(I I I, I V)$ intersect at right angles because of the local exponent differences $1 / 2$ at the finite singularities. In particular, let us choose $y_{1}$ such that it is the holomorphic solution around $z_{2}$ whose powerseries has constant term 1 and let $y_{2}$ be the unique solution starting with $\left(z-z_{2}\right)^{1 / 2}(1+\cdots)$. Then a possible image of the Schwarz map looks like

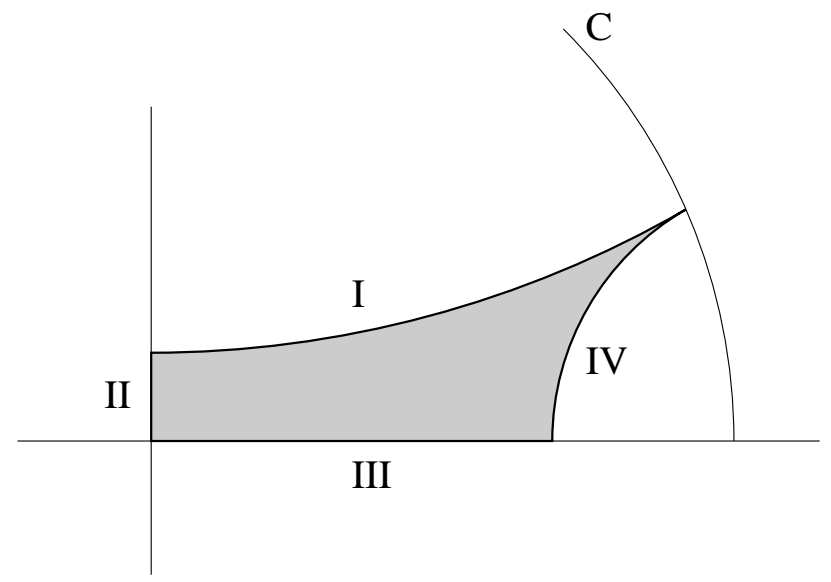

However, one should be careful with pictures like this. The above image corresponds to the situation when $y_{1}$ has no zeros on the intervals $\left(z_{1}, z_{2}\right)$ and $\left(z_{2}, z_{3}\right)$. When $y_{1}$ does have zeros on these intervals, the image of the Schwarz map may overlap itself several times.

Let $\bar{S}$ be the group generated by the complex reflections in the four circles. Let $S \subset \bar{S}$ be the subgroup of index two of automorphisms of $\mathbb{P}^{1}$. Then it also well-known that the monodromy group $G$ modulo scalars is precisely $S$.

Clearly there is a circle $C$ with 0 as origin, which passes through the point of tangency between $I, I V$. The following Lemma is more or less obvious.

Lemma 1.2 The group $G$ is unitary if and only if $C$ is orthogonal to the circle segments $I, I V$.

The question of the orthogonality of circles like $C$ was asked by F.Klein in [Kl1907]. Based on the so-called oscillation theorems of Hilbert it can be established that there exists an infinite, but discrete, set of real values of $B$ for which $C$ is indeed orthogonal. At that time this result was considered a possible step towards the solution of the uniformisation problem.

Very soon afterwards, E.Hilb, [Hi1909] established a similar theorem for differential equation with four singularities and general exponents.

Now that the uniformisation problem has been solved by methods from analysis, we can reverse the situation and show the existence of at least one $B$ for which (1) has unitary monodromy. Let $D$ be the hyperbolic disc and $j: D \rightarrow \mathbb{C} \backslash\left\{z_{1}, z_{2}, z_{3}\right\}$ be the universal cover of $\mathbb{P}^{1}$ minus the points $z=z_{1}, z_{2}, z_{3}, \infty$. Apply the Schwarzian derivative

$$
S: \eta(z) \mapsto \frac{\eta^{\prime \prime \prime}}{\eta^{\prime \prime}}-\frac{3}{2}\left(\frac{\eta^{\prime \prime}}{\eta^{\prime}}\right)^{2}
$$


Then $S\left(j^{-1}\right)$ is a rational function $Q$ of $z$ regular outside of $z_{1}, z_{2}, z_{3}, \infty$ and poles of order at most two. It is known that $u^{\prime \prime}+Q u=0$ is a Fuchsian equation of order two such that there exist two solutions $u_{1}, u_{2}$ with the property that $j\left(u_{1} / u_{2}\right)=z$. Replacing $u$ by $\sqrt{4 z^{3}-g_{2} z-g_{3}} y$, the equation changes into an equation of type (1). The projectivised monodromy of this equation is precisely the covering group of our universal cover.

It is the purpose of this paper to rewrite the unitarity problem of $G$ as a spectral problem in the space of real-analytic functions on $\mathbb{C} \backslash\left\{z_{1}, z_{2}, z_{3}\right\}$ with the values of $B$ as spectrum. Moreover, an asymptotic analysis of the spectrum indicates that eigenvalues to the problem occur in abundance. See Conjecture 5.1 and Theorem 7.1. In particular we see that most eigenvalues $B$ are complex numbers.

\section{Unitary groups}

Let $H$ be a Hermitean matrix with $\operatorname{det}(H) \neq 0$. We define the corresponding unitary group $U(H)$ by

$$
U(H)=\left\{g \in G L(2, \mathbb{C}) \mid \bar{g}^{t} H g=H\right\} .
$$

Notice that for any $h \in G L(2, \mathbb{C})$ we have

$$
U\left(\bar{h}^{t} H h\right)=h^{-1} U(H) h .
$$

We can conjugate the group $U(H)$ in such a way that the corresponding Hermitian matrix is either $\left(\begin{array}{ll}1 & 0 \\ 0 & 1\end{array}\right)$ or $\left(\begin{array}{cc}1 & 0 \\ 0 & -1\end{array}\right)$. In the first case we call $H$, and its conjugated versions, positive definite, in the second case indefinite.

In this paper we shall deal with indefinite Hermitean forms. In particular we take the form

$$
H_{0}=\left(\begin{array}{cc}
0 & i \\
-i & 0
\end{array}\right)
$$

as standard Hermitean form.

Proposition 2.1 The unitary group $U\left(H_{0}\right)$ is the group generated by $S L(2, \mathbb{R})$ and the diagonal matrices $\lambda \mathrm{I}_{2}$ with $\lambda \in \mathbb{C},|\lambda|=1$.

Proof. Suppose $g=\left(\begin{array}{ll}a & b \\ c & d\end{array}\right) \in U\left(H_{0}\right)$. Then, from $\bar{g}^{t} H_{0} g=H_{0}$ it follows that

$$
\left(\begin{array}{ll}
\bar{a} c-a \bar{c} & \bar{a} d-b \bar{c} \\
\bar{b} c-a \bar{d} & \bar{b} d-b \bar{d}
\end{array}\right)=\left(\begin{array}{cc}
0 & 1 \\
-1 & 0
\end{array}\right)
$$

The first case we look at is when $a=0$. In that case we get $-b \bar{c}=1$ and $\bar{b} d \in \mathbb{R}$. Note that $b \neq 0$. Choose $\beta \in \mathbb{R}$ and $b^{*} \in \mathbb{C}$ with $\left|b^{*}\right|=1$ such that $b=\beta b^{*}$. From $\bar{b} d \in \mathbb{R}$ it follows that $d$ is a real multiple of $b$. So $d=\delta b^{*}$ where $\delta \in \mathbb{R}$. Similarly it follows from the first equation that $c=\gamma b^{*}$ for some $\gamma \in \mathbb{R}$. More particularly, $-b \bar{c}=1$ implies that $-\beta \gamma=1$. So we see that

$$
g=b^{*}\left(\begin{array}{cc}
0 & \beta \\
\gamma & \delta
\end{array}\right) \quad \text { with }\left|b^{*}\right|=1 \text { and }-\gamma \beta=1
$$


This proves our assertion when $a=0$.

Similarly we deal with the case $d=0$. Let us now assume that $a, d \neq 0$. Put $a=\alpha a^{*}$ with $\alpha \in \mathbb{R}$ and $\left|a^{*}\right|=1$. From $\bar{a} c-a \bar{c}=0$ it follows that $\bar{a} c \in \mathbb{R}$. Hence $c$ is a real multiple of $a$. Put $c=\gamma a^{*}$ for some $\gamma \in \mathbb{R}$. Similarly it follows from $\bar{b} d-b \bar{d}=0$ that $b$ is a real multiple of $d$, say $b=\lambda d$ with $\lambda \in \mathbb{R}$. Lastly it follows from $\bar{a} d-b \bar{c}=1$ that $\bar{a} d(1-\gamma \lambda)=1$. We now conclude that $d$ is a real multiple of $a$. Let us now put $d=\delta a^{*}$. Then $\bar{a} d-b \bar{c}=1$ implies that

$$
1=(\alpha \delta-\beta \gamma)\left|a^{*}\right|^{2}=\alpha \delta-\beta \gamma
$$

Hence

$$
g=a^{*}\left(\begin{array}{cc}
\alpha & \beta \\
\gamma & \delta
\end{array}\right) \quad \alpha \delta-\gamma \beta=1
$$

as asserted.

qed

\section{Monodromy groups}

In this section we gather some information on the monodromy representation corresponding to (1). Fix a base point $z_{0}$ in the complex plane and let $\Gamma_{1}, \Gamma_{2}, \Gamma_{3}$ be simple closed loops beginning and ending in $z_{0}$ which encircle respectively the points $z_{1}, z_{2}, z_{3}$ counter clockwise. Let $M_{1}, M_{2}, M_{3}$ be the corresponding local monodromy matrices. The local monodromy matrix $M_{\infty}$ around infinity is given by the relation $M_{1} M_{2} M_{3} M_{\infty}=$ Id. Note that the finite local monodromies have eigenvalues $1,-1$, hence $M_{1}^{2}=M_{2}^{2}=M_{3}^{2}=\operatorname{Id}$ and $M_{1}, M_{2}, M_{3}$ are reflections. The matrix $M_{\infty}$ has coinciding eigenvalues $i, i$ or $-i,-i$ and, consequently, trace $\pm 2 i$. It cannot be a scalar since we always have logarithmic solutions around $z=\infty$. So we conclude that $M_{\infty}$ is a parabolic element (in this paper a scalar element is not considered to be parabolic). Let $G$ be the group generated by these local monodromies.

A first remark we like to make is that $G$ acts irreducibly on the space of solutions. Suppose on the contrary that $G$ acts reducibly. Then $M_{1}, M_{2}, M_{3}, M_{\infty}$ have a common eigenvector $v$. Let $\lambda_{1}, \lambda_{2}, \lambda_{3}, \lambda_{\infty}$ be the corresponding eigenvalues. Their product should be one. But this is impossible since $\lambda_{\infty}=i$ and the other eigenvalues are \pm 1 . So we conclude that $G$ acts irreducibly. The irreducibility of $G$ also implies that any $G$-invariant hermitian form is non-degenerate and uniquely determined up to scalars.

In the following Proposition we give necessary and sufficient conditions for the unitarity of the group generated by three involutions whose product is parabolic.

Proposition 3.1 Let $P, Q, R \in G L(2, \mathbb{C})$ be reflections (eigenvalues $1,-1$ ) and suppose that $P Q R$ is parabolic with trace $\pm 2 i$. Let $G$ be the group generated by $P, Q, R$ and denote by $t_{M}$ the trace of a $2 \times 2$-matrix $M$. Then the following statements are equivalent

1. $G$ is unitary

2. $t_{P Q}, t_{Q R}, t_{P R}$ are real.

3. $t_{P Q}$ and $t_{Q R}$ are real and satisfy $\left(t_{P Q}^{2}-4\right)\left(t_{Q R}^{2}-4\right) \geq 16$.

In the proof the following Lemma is useful. 
Lemma 3.2 Let $P, Q, R \in G L(2, \mathbb{C})$ be reflections (eigenvalues $1,-1$ ). Then

$$
t_{P Q}^{2}+t_{Q R}^{2}+t_{P R}^{2}-t_{P Q} t_{Q R} t_{P R}=2+t_{(P Q R)^{2}} .
$$

Suppose in addition that $P Q R$ is parabolic with trace $\pm 2 i$ and $t_{P Q}, t_{Q R}, t_{P R} \in \mathbb{R}$. Then $\left|t_{P Q}\right|,\left|t_{Q R}\right|,\left|t_{P R}\right|>2$.

Proof. The identity

$$
t_{P Q}^{2}+t_{Q R}^{2}+t_{P R}^{2}-t_{P Q} t_{Q R} t_{P R}=2+t_{(P Q R)^{2}}
$$

can be proven by a straightforward computation. Suppose $P Q R$ is parabolic with trace $\pm 2 i$, the matrix $(P Q R)^{2}$ is parabolic with trace -2 . Hence

$$
t_{P Q}^{2}+t_{Q R}^{2}+t_{P R}^{2}-t_{P Q} t_{Q R} t_{P R}=0 .
$$

Supppose al three traces in this equation are real. Consider the equation as a quadratic equation in $t_{P Q}$. Then its discriminant should be $\geq 0$. This means, $\left(t_{P R} t_{Q R}\right)^{2}-4\left(t_{P R}^{2}+t_{Q R}^{2}\right) \geq$ 0 and hence

$$
\left(t_{P R}^{2}-4\right)\left(t_{Q R}^{2}-4\right) \geq 16 .
$$

Similar inequalities hold for any other pair of traces. The inequalities imply that either the absolute values of all traces are $>2$, as asserted, or that all traces are zero.

In the latter case we consider the group $G$ generated by $P, Q, R$ in more detail. Since the trace of $P Q$ is zero and determinant 1 , the eigenvalues of $P Q$ are $\pm i$. Hence $(P Q)^{2}=-\mathrm{Id}$. Using this and $P^{2}=Q^{2}=$ Id we get $P Q=-Q P$. Similarly for the other pairs. From this we conclude that $G$ modulo scalars is an abelian group of order 4 . Hence $G$ is a finite group and $P Q R$ cannot be parabolic, since parabolic elements have infinite order.

qed

Proof of Proposition 3.1. From the proof of Lemma 3.2 we see that $t_{P Q}, t_{Q R}, t_{R P} \in \mathbb{R}$ is equivalent to $t_{P Q}, t_{Q R} \in \mathbb{R}$ and $\left(t_{P Q}^{2}-4\right)\left(t_{Q R}^{2}-4\right) \geq 16$. Hence it remains to show the equivalence of (1) and (2).

First we prove $(1) \Rightarrow(2)$. So suppose that $G$ is unitary. Since $G$ contains the parabolic element $P Q R$ the signature of the hermitian form should be $(1,1)$. Without loss of generality we may then assume that the hermitian form is given by $H_{0}$ as defined in the previous section. Since $P, Q, R$ are determinant -1 matrices, it follows from Proposition 2.1 that they should be of the form $i N$, where $N \in S L(2, \mathbb{R})$. Hence $P Q, Q R, P R \in S L(2, \mathbb{R})$ and so their traces are real.

Proof of $(2) \Rightarrow(1)$. Suppose that $t_{P Q}, t_{Q R}, t_{P R}$ are real. Since $P Q R$ is parabolic with trace $\pm 2 i$, Lemma 3.2 tells us that all traces have absolute value $>2$. By conjugation we can see to it that

$$
P=\left(\begin{array}{cc}
0 & i \\
-i & 0
\end{array}\right)
$$

Suppose that $Q=\left(\begin{array}{cc}p & q \\ r & -p\end{array}\right)$. Choose $a, b \in \mathbb{C}$ such that $a^{2}+b^{2}=1$ and $\left(a^{2}-b^{2}\right) p+a b(q+r)=$ 0 . Then conjugation by $M=\left(\begin{array}{cc}a & b \\ -b & a\end{array}\right)$ leaves $P$ fixed and changes $Q$ into

$$
M Q M^{-1}=\left(\begin{array}{cc}
0 & -2 a b p+a^{2} q-b^{2} r \\
-2 a b p-b^{2} q+a^{2} r & 0
\end{array}\right) .
$$


Adopting this conjugation we get a new $Q=\left(\begin{array}{cc}0 & q^{\prime} \\ r^{\prime} & 0\end{array}\right)$ with $q^{\prime} r^{\prime}=1$. From the fact that $t_{P Q} \in \mathbb{R}$ it follows that $i\left(q^{\prime}-r^{\prime}\right) \in \mathbb{R}$. Put $q^{\prime}=i \lambda$. Substitute this in $q^{\prime} r^{\prime}=1$ to find that $r^{\prime}=$ $-i / \lambda$. Now $i\left(q^{\prime}-r^{\prime}\right) \in \mathbb{R}$ implies that $\lambda+1 / \lambda \in \mathbb{R}$. We also know that $|\lambda+1 / \lambda|=\left|t_{P Q}\right|>2$. Hence $\lambda \in \mathbb{R}$, and we conclude

$$
Q=i\left(\begin{array}{cc}
0 & \lambda \\
-1 / \lambda & 0
\end{array}\right) \in i S L(2, \mathbb{R})
$$

Now put $R=\left(\begin{array}{cc}p & q \\ r & -p\end{array}\right)$ for some suitable $p, q, r \in \mathbb{C}$. Notice that $t_{P R}=i(q-r)$ and $t_{Q R}=i(p \lambda-r / \lambda)$. Solving for $q, r$ gives

$$
q=i \frac{t_{P R} \lambda-t_{Q R}}{\lambda-1 / \lambda} \quad r=i \frac{t_{P R} / \lambda-t_{Q R}}{\lambda-1 / \lambda} .
$$

In particular we see that $q, r$ are purely imaginary. The determinant of $R$ is -1 . In other words, $-p^{2}-q r=-1$. Hence we find

$$
\begin{aligned}
-p^{2} & =q r-1=-\frac{\left(t_{P R} \lambda-t_{Q R}\right)\left(t_{P R} / \lambda-t_{Q R}\right)}{(\lambda-1 / \lambda)^{2}}-1 \\
& =-\frac{t_{P R}^{2}+t_{Q R}^{2}-t_{P Q} t_{P R} t_{Q R}}{(\lambda-1 / \lambda)^{2}}-1
\end{aligned}
$$

In the last line we used $\lambda+1 / \lambda=t_{P Q}$. We now use the trace identity of Lemma 3.2 to find

$$
\begin{aligned}
-p^{2} & =\frac{t_{P Q}^{2}}{(\lambda-1 / \lambda)^{2}}-1 \\
& =\frac{(\lambda+1 / \lambda)^{2}}{(\lambda-1 / \lambda)^{2}}-1=\frac{4}{(\lambda-1 / \lambda)^{2}}
\end{aligned}
$$

Hence

$$
p=\frac{2 i}{(\lambda-1 / \lambda)}=\frac{2 i}{\sqrt{t_{P Q}^{2}-4}} .
$$

In particular $p$ is purely imaginary. So we conclude that $R$ is $i$ times a matrix from $S L(2, \mathbb{R})$. Now we see that our normalised $P, Q, R$ have the standard form $H_{0}$ as common invariant form.

qed

\section{A spectral problem}

We remind the reader of the concept of Wronskian determinant of a second order equation $y^{\prime \prime}+p y^{\prime}+q y=0$ where $p, q$ are analytic functions in a simply connected domain $U \subset \mathbb{C}$. Let $y_{1}, y_{2}$ be two independent solutions and consider $W=y_{1}^{\prime} y_{2}-y_{2}^{\prime} y_{1}$. It is easy to see that $W$ satisfies the differential equation $W^{\prime}=-p W$, hence $W=\alpha \exp \left(-\int p d z\right)$ for some non-zero constant $\alpha$. In particular $W$ has no zeros or poles in $U$. 
In what follows we shall also take into account real analytic solutions of our differential equation. Since such functions are not holomorphic we must replace the complex differentiation operator $\frac{d}{d z}$ by its real counterpart. Let us therefore rewrite our second order equation in the form

$$
\partial_{z}^{2} y+p \partial_{z} y+q y=0
$$

where

$$
\partial_{z}=\frac{1}{2}\left(\frac{\partial}{\partial x}-i \frac{\partial}{\partial y}\right)
$$

In the following Proposition we show that the real solution space is a four dimensional $\mathbb{R}$-vector space.

Proposition 4.1 Let $U$ be as above. Let $y_{1}, y_{2}$ be two independent complex analytic solutions of (2) in $U$. Then the $\mathbb{R}$-vector space of real $C^{2}$ solutions in $U$ is four dimensional and spanned by $y_{1} \bar{y}_{1}, \operatorname{Re}\left(y_{1} \bar{y}_{2}\right), \operatorname{Im}\left(y_{1} \bar{y}_{2}\right), y_{2} \bar{y}_{2}$.

We denote the real valued $C^{2}$-functions on $U$ by $C^{2}(U, \mathbb{R})$ and the complex-valued ones by $C^{2}(U, \mathbb{C})$.

Proof. Let $u$ be any real $C^{2}$-solution on $U$ of (2). Choose $A, B \in C^{2}(U, \mathbb{C})$ such that

$$
\begin{aligned}
u & =A y_{1}+B y_{2} \\
\partial_{z} u & =A y_{1}^{\prime}+B y_{2}^{\prime}
\end{aligned}
$$

We do this by solving for $A, B$. We find

$$
\begin{aligned}
& A=\left(y_{2}^{\prime} u-y_{2} \partial_{z} u\right) / W \\
& B=\left(-y_{1}^{\prime} u+y_{1} \partial_{z} u\right) / W
\end{aligned}
$$

In particular, $A, B \in C^{2}(U)$ since the Wronskian $W$ has no zeros in $U$. A simple calculation shows that $\partial_{z} A=\partial_{z} B=0$, hence $A, B$ are anti-holomorphic functions, i.e. $\bar{A}, \bar{B}$ are holomorphic. Let us rewrite (2) in the form $M y=0$, where $M=\partial_{z}^{2}+p \partial_{z}+q$. Note that $M \bar{u}=0$ since $u$ is real. After substitution of $u=A y_{1}+B y_{2}$ this yields

$$
0=(M \bar{A}) \overline{y_{1}}+(M \bar{B}) \overline{y_{2}} .
$$

Since $\overline{\partial_{z}}$ commutes with any holomorphic differential operator we get $M \overline{\partial_{z} u}=\overline{\partial_{z}} M \bar{u}=0$. Hence

$$
0=(M \bar{A}) \overline{y_{1}^{\prime}}+(M \bar{B}) \overline{y_{2}^{\prime}} .
$$

Solving equations (3) and (4) yields $M \bar{A}=M \bar{B}=0$. So $\bar{A}$ and $\bar{B}$ are holomorphic functions satisfying $M y=0$. Hence there exist complex constants $\alpha, \beta, \gamma, \delta$ such that $A=\alpha \overline{y_{1}}+\beta \overline{y_{2}}$ and $B=\gamma \overline{y_{1}}+\delta \overline{y_{2}}$ on $U$. We conclude that

$$
u=\alpha y_{1} \overline{y_{1}}+\beta y_{1} \overline{y_{2}}+\gamma y_{2} \overline{y_{1}}+\delta y_{2} \overline{y_{2}} .
$$

Hence we see that our solution space is spanned by the functions of our Proposition. 
It remains to show that the four functions $y_{i} \overline{y_{j}}$ are $\mathbb{C}$-linear independent. Suppose that there exist $\alpha, \beta, \gamma, \delta$ such that

$$
0=\alpha y_{1} \overline{y_{1}}+\beta y_{1} \overline{y_{2}}+\gamma y_{2} \overline{y_{1}}+\delta y_{2} \overline{y_{2}} .
$$

Apply the operator $\partial_{z}$,

$$
0=\alpha y_{1}^{\prime} \overline{y_{1}}+\beta y_{1}^{\prime} \overline{y_{2}}+\gamma y_{2}^{\prime} \overline{y_{1}}+\delta y_{2}^{\prime} \overline{y_{2}} .
$$

These two inequalities together yield $\alpha \overline{y_{1}}+\beta \overline{y_{2}}=0$ and $\gamma \overline{y_{1}}+\delta \overline{y_{2}}=0$. Since $y_{1}, y_{2}$ are independent functions this implies $\alpha=\beta=\gamma=\delta=0$. Hence our functions are indeed independent.

qed

Proposition 4.2 Let $G$ be the monodromy group of the linear second order differential equation $y^{\prime \prime}+p y^{\prime}+q y=0$ where $p, q \in \mathbb{C}(z)$. Let $S$ be the set of poles of $p$ and $q$. Then $G$ is unitary if and only if there exists a non-trivial real, $C^{2}$ function $f$ on $\mathbb{C} \backslash S$ which is a solution of (2). Moreover, this function $f$ is uniquely determined up to a constant factor.

Proof. Suppose first that $G$ is unitary. Hence there exists a non-trivial $2 \times 2$-matrix $H$ so that $\bar{H}^{t}=H$ and $\bar{g}^{t} H g=H$ for all $g \in G$. The bar denotes complex conjugation and the superscript ${ }^{t}$ denotes transposition of a matrix. Let $y_{0}, y_{1}$ be any two independent solutions around a non-singular point $z_{0}$. Then we see that $f=\left(\bar{y}_{1}, \bar{y}_{2}\right) H\left(\begin{array}{l}y_{1} \\ y_{2}\end{array}\right)$ is invariant under monodromy. Hence $f$ can be extended globally throughout $\mathbb{C} \backslash S$. Moreover it is real-valued and real-analytic. Furthermore, $f$ cannot be identically zero since the functions $y_{i} \overline{y_{j}}$ are linearly independent according to Proposition 4.1. Finally, since $\partial_{z} \bar{y}_{1}=\partial_{z} \bar{y}_{2}=0$ we see that $f$ satisfies our equation (2).

Suppose conversely that we have a global real, $C^{2}$-function $f$ satisfying equation (2). Choose a simply connected domain $U \in \mathbb{C} \backslash S$ and two independent complex analytic solutions $y_{1}, y_{2}$ of (2), defined on $U$. In Proposition 4.1 we have seen that there exist unique numbers $\alpha, \beta, \gamma, \delta$ such that

$$
f=\alpha y_{1} \overline{y_{1}}+\beta y_{1} \overline{y_{2}}+\gamma y_{2} \overline{y_{1}}+\delta y_{2} \overline{y_{2}} .
$$

Hence

$$
f=\left(\overline{y_{1}}, \overline{y_{2}}\right) H\left(\begin{array}{l}
y_{1} \\
y_{2}
\end{array}\right)
$$

where $H=\left(\begin{array}{ll}\alpha & \beta \\ \gamma & \delta\end{array}\right)$. Since $f$ is real-valued, conjugation and transposition show that $\bar{H}^{t}=H$. Furthermore, $f$ is globally defined, hence $\bar{g}^{t} H g=H$ for any $g \in G$.

Finally the existence two independent global real solutions to 2 would imply the existence of two independent $G$-invariant Hermitian forms. However this is impossible in view of the irreducible action of $G$. Therefore $f$ is uniquely determined up to scalars.

qed

Let us now return to our equation (1) and its monodromy group $G$. Note that Proposition 4.2 has now turned the unitary problem of $G$ into the problem

$$
L f=B f, \quad f \in C^{2}\left(\mathbb{C} \backslash\left\{z_{1}, z_{2}, z_{3}\right\}, \mathbb{R}\right)
$$

where

$$
L=P \partial_{z}^{2}+\left(P^{\prime} / 2\right) \partial_{z}+z / 4
$$


The interesting point here is that we now restated our unitarity problem as an eigenvalue problem on $C^{2}\left(\mathbb{C} \backslash\left\{z_{1}, z_{2}, z_{3}\right\}\right)$. In particular, the eigenspace for any dimension is at most one by the uniqueness (up to scalars) of $f$. It would be of great interest to determine the complete spectrum.

To initiate this study we rewrite equation (1) using elliptic functions. As is well known the elliptic curve $E: y^{2}=4 x^{3}-g_{2} x-g_{3}$ can be parametrised by a suitable Weierstrass $\wp$-function as follows, $x=\wp(z), y=\wp^{\prime}(z)$. Replace $z$ by $\wp(z)$ in (1) to get

$$
\frac{d^{2} y}{d z^{2}}+\left(\frac{\wp(z)}{4}-B\right) y=0 .
$$

We denote the period lattice of $E$ by $\Lambda$. The Lamé equation can thus be considered either as a differential equation in $\mathbb{C}$ with doubly periodic coefficients and singularities in $\Lambda$, or a differential equation on the elliptic curve $E$ with a single singularity at the point $\infty$ of $E$. In both cases the local exponents at the singularity read $1 / 2,1 / 2$. The fundamental group of $E$ minus $\infty$ is the free group on two generators, where the generators are formed by a basis $\gamma_{1}, \gamma_{2}$ of the closed paths on $E$. In the covering space $\mathbb{C} \rightarrow \mathbb{C} / \Lambda=E$ these closed loops correspond to two periods $\omega_{1}, \omega_{2} \in \Lambda$ say. The commutator $\gamma_{1} \gamma_{2} \gamma_{1}^{-1} \gamma_{2}^{-1}$ is the closed simple path around the point $\infty$ on $E$. Since the local exponents there are equal and $1 / 2$, the local monodromy matrix at $\infty$ is parabolic with trace -2 . The monodromy group $H$ of (6) is generated by the subgroup of $G$ consisting of the determinant 1 elements. So, with the above notations $H=\left\langle M_{1} M_{2}, M_{2} M_{3}\right\rangle$. for example. In particular $H$ has index two in $G$.

The spectral problem (5) can now be lifted to

$$
\partial_{z}^{2} u+\frac{\wp(z)}{4} u=B u, \quad u \in C^{2}(\mathbb{C} \backslash \Lambda), u \text { real }- \text { valued and even }
$$

The extra condition that $u$ is even (i.e. $u(-z)=u(z)$ ) is a remnant of the fact that $u$ is a pullback via the covering map of $E$ from the original Lamé spectral problem (5).

\section{$5 \quad$ Asymptotic analysis}

Instead of looking at the spectral problem (7) we consider the more general version

$$
\partial_{z}^{2} u-n(n+1) \wp(z) u=B u, \quad u \in C^{2}(\mathbb{C} \backslash \Lambda), u \text { real }- \text { valued and even }
$$

which corresponds to the general Lamé equation with parameter $n$. We take $n \in \mathbb{R}$. The problem (7) corresponds to the choice $n=-1 / 2$.

Let $\Lambda$ be the lattice corresponding to the elliptic curve $y^{2}=4 x^{3}-g_{2} x-g_{3}$ and let $\omega_{1}, \omega_{2}$ be a $\mathbb{Z}$-basis satisfying $\operatorname{Im}\left(\omega_{2} / \omega_{1}\right)>0$.

By $\eta_{1}, \eta_{2}$ we denote the quasi-periods corresponding to the lattice $\Lambda$. They are defined by $\eta_{i}=\zeta\left(z+\omega_{i}\right)-\zeta(z)$ where $\zeta(z)$ is the Weierstrass $\zeta$-function defined by $\zeta^{\prime}(z)=-\wp(z)$ and $\zeta(z)=-\zeta(-z)$. We let $\Delta=\left(\omega_{2} \overline{\omega_{1}}-\omega_{1} \overline{\omega_{2}}\right) / 2 i$. Note that $\Delta$ is real and positive because $\operatorname{Im}\left(\omega_{2} / \omega_{1}\right)>0$. Moreover, $\Delta$ is the area of a fundamental paralellogram of $\Lambda$. We also define $a=\eta_{1} \overline{\omega_{2}}-\eta_{2} \overline{\omega_{1}}$.

In this section we provide evidence in the form of a perturbation calculation for the validity of the following conjecture. 
Conjecture 5.1 Let notations be as above. Up to order $1 /\left|l_{0}\right|$ the values of $B$ in problem (7) are given by

$$
B=l_{0}^{2}-n(n+1) \frac{a}{2 i \Delta}+\frac{\pi}{4 \Delta} \frac{l_{0}}{\overline{l_{0}}}
$$

where $l_{0} \in \bar{\Lambda}$, the lattice generated by $\overline{\omega_{2}}, \overline{\omega_{1}}$.

Let us first solve (8) when $n(n+1)$ is replaced by 0 and $B$ is replaced by $l^{2}$ for convenience. The real-valued solutions of $\partial_{z}^{2} u=l^{2} u$ are given by

$$
A e^{l z+\overline{l z}}+B\left(e^{l z-\overline{l z}}+e^{-l z+\overline{l z}}\right)+\frac{C}{i}\left(e^{l z-\overline{l z}}-e^{-l z+\overline{l z}}\right)+D e^{-l z-\overline{l z}}
$$

with $A, B, C, D \in \mathbb{R}$. The condition that $u$ is even sees to it that $C=0$ and $A=D$, so we get

$$
u=A\left(e^{l z+\overline{l z}}+e^{-l z-\overline{l z}}\right)+B\left(e^{l z-\overline{l z}}+e^{-l z+\overline{l z}}\right) .
$$

If $A \neq 0$ we see that $u$ cannot be periodic with respect to $\Lambda$. So $A=0$ and periodicity of $u$ now implies the existence of $m_{1}, m_{2} \in \mathbb{Z}$ such that

$$
\begin{aligned}
& l \omega_{1}-\overline{l \omega_{1}}=-2 \pi i m_{2} \\
& l \omega_{2}-\overline{l \omega_{2}}=2 \pi i m_{1}
\end{aligned}
$$

Hence $l=\pi\left(m_{2} \overline{\omega_{2}}+m_{1} \overline{\omega_{1}}\right) / \Delta$.

Let us now turn to an asymptotic study of the original problem (8) with $B=l^{2}$ when $|l| \rightarrow \infty$. We first find an approximate solution of the complex differential equation $u^{\prime \prime}-n(n+1) \wp u=l^{2} u$. Put $u=e^{l z+\beta(z)}$ for some $\beta(z)$. We find, $\beta^{\prime \prime}+\left(\beta^{\prime}\right)^{2}+2 l \beta^{\prime}-n(n+1) \wp(z)=0$. Now consider the asymptotic expansion

$$
\beta(z)=\frac{\beta_{1}(z)}{l}+\frac{\beta_{2}(z)}{l^{2}}+\cdots
$$

After substitution into the differential equation and comparison of equal powers of $1 / l$ we can find the $\beta_{k}$ recursively as follows,

$$
\begin{aligned}
2 \beta_{1}^{\prime}-n(n+1) \wp & =0 \\
2 \beta_{2}^{\prime}+\beta_{1}^{\prime \prime} & =0 \\
2 \beta_{3}^{\prime}+\beta_{1}^{\prime} \beta_{2}^{\prime}+\beta_{2}^{\prime \prime} & =0
\end{aligned}
$$

We shall consider the second order approximation of $\beta$. Denote by $\zeta(z)$ the Weierstrass $\zeta$ function, i.e. $\zeta^{\prime}(z)=-\wp(z)$. Then, $\beta_{1}(z)=-n(n+1) \zeta(z) / 2$. From the second equation we infer $\beta_{2}(z)=-\beta_{1}^{\prime}(z) / 2=-n(n+1) \wp(z) / 4$.

Let us first compute the spectrum up to order $1 /|l|$. We perturb the solution $e^{l z-\overline{l z}}+$ c.c. to

$$
\exp (l z-n(n+1) \zeta(z) / 2 l-\overline{l z}+\overline{n(n+1) \zeta(z)} / 2 \bar{l})+c . c .
$$


Here $c . c$ stands for complex conjugate. The periodicity conditions now imply the existence of $m_{1}, m_{2} \in \mathbb{Z}$ such that

$$
\begin{aligned}
& l \omega_{1}-\overline{l \omega_{1}}-n(n+1) \frac{\eta_{1}}{2 l}+n(n+1) \frac{\overline{\eta_{1}}}{2 \bar{l}}=-2 \pi i m_{2}+O\left(1 /|l|^{2}\right) \\
& l \omega_{2}-\overline{l \omega_{2}}-n(n+1) \frac{\eta_{2}}{2 l}+n(n+1) \frac{\overline{\eta_{2}}}{2 \bar{l}}=2 \pi i m_{1}+O\left(1 /|l|^{2}\right)
\end{aligned}
$$

where $\eta_{i}=\zeta\left(z+\omega_{i}\right)-\zeta(z)$ for $i=1,2$. Put $l_{0}=\pi\left(m_{2} \overline{\omega_{2}}+m_{1} \overline{\omega_{1}}\right) / \Delta$ and $l=l_{0}+\epsilon$. Then, up to second order in $1 / l_{0}$,

$$
\begin{aligned}
& \epsilon \omega_{1}-\overline{\epsilon \omega_{1}}=n(n+1) \eta_{1} / 2 l_{0}-c . c \\
& \epsilon \omega_{2}-\overline{\epsilon \omega_{2}}=n(n+1) \eta_{2} / 2 l_{0}-c . c
\end{aligned}
$$

Solution of $\epsilon$ yields

$$
l=l_{0}-\frac{n(n+1)}{2 i \Delta}\left(\frac{a}{l_{0}}+\frac{b}{\overline{l_{0}}}\right)+O\left(\frac{1}{\left|l_{0}\right|^{2}}\right)
$$

where $a=\left(\eta_{1} \overline{\omega_{2}}-\eta_{2} \overline{\omega_{1}}\right)$ and $b=\left(\overline{\eta_{2} \omega_{1}}-\overline{\eta_{1} \omega_{2}}\right)$. Notice that $b$ equals $2 \pi i$ because of Legendre's relation. We conclude,

$$
B=l^{2}=l_{0}^{2}-\frac{n(n+1)}{2 i \Delta}\left(a+2 \pi i \frac{l_{0}}{\bar{l}_{0}}\right)+O\left(\frac{1}{\left|l_{0}\right|}\right),
$$

as conjectured.

In the Section 6 we shall see that the agreement with numerical data in the case $n=-1 / 2$ is remarkably well. In Section 7 we give a proof with convergent series in the case when $n=1$.

\section{Numerical data}

Now let us take $n=-1 / 2$, which is the original problem. The generators of the monodromy of equation (1) can be computed numerically as follows. Fix a non-singular point $a_{0}$ and construct three simple loops, each enclosing one of the finite singularities exactly once. Let $\gamma$ be such a loop. We discretize $\gamma$ by choosing $N$ points $a_{0}, a_{1}, a_{2}, \ldots, a_{N}=a_{0}$ on $\gamma$ which are regularly spaced and whose increasing indices follow the orientation of $\gamma$. Now write equation (1) as a first order system

$$
\begin{aligned}
y^{\prime} & =v \\
v^{\prime} & =-\frac{P^{\prime}}{2 P} v-\frac{z / 4-B}{2 P} y
\end{aligned}
$$

and solve this system numerically with a Runge-Kutta method (we used fourth order) using the points $a_{i}$. As initial column vectors we chose $(1,0)^{t}$ and $(0,1)^{t}$. After numerical integration we obtain two column vectors $(a, c)^{t}$ and $(b, d)^{t}$. The monodromy matrix corresponding to $\gamma$ is then approximated by $\left(\begin{array}{ll}a & b \\ c & d\end{array}\right)$.

We can now compute $M_{1}, M_{2}, M_{3}$ to any accuracy we like, by increasing the number of interpolation points $a_{i}$. In order to determine $B$ such that the monodromy group is unitary 
we use Proposition 3.1 and interpolation. For any choice of $B$ we can compute the traces $t_{12}:=t_{M_{1} M_{2}}$ and $t_{23}:=t_{M_{2} M_{3}}$. These are complex analytic functions of $B$. Choose a value $B_{0}$ of $B$ and a nearby value $B_{1}$. The derivative of $t_{12}, t_{23}$ at $B_{0}$ can be approximated by

$$
\lambda=\frac{t_{12}\left(B_{1}\right)-t_{12}\left(B_{0}\right)}{B_{1}-B_{0}} \quad \text { and } \quad \mu=\frac{t_{23}\left(B_{1}\right)-t_{23}\left(B_{0}\right)}{B_{1}-B_{0}} .
$$

The linear approximations of $t_{12}, t_{23}$ at $B=B_{0}$ now read $t_{12}(B)=t_{12}\left(B_{0}\right)+\lambda\left(B-B_{0}\right)$ and $t_{23}(B)=t_{23}\left(B_{0}\right)+\mu\left(B-B_{0}\right)$. We now solve $x \in \mathbb{C}$ from the system of equations

$$
\operatorname{Im}\left(t_{12}\left(B_{0}\right)+\lambda x\right)=0, \quad \operatorname{Im}\left(t_{23}\left(B_{0}\right)+\mu x\right)=0 .
$$

A brief computation gives us

$$
x=\frac{\bar{\mu} \operatorname{Im}\left(t_{12}\left(B_{0}\right)\right)-\bar{\lambda} \operatorname{Im}\left(t_{23}\left(B_{0}\right)\right)}{\operatorname{Im}(\bar{\lambda} \mu)} .
$$

Supposedly the value $B_{0}+x$ should be a closer approximation to a spectral value of $B$ than $B_{0}$. We then repeat the argument with the new value of $B$. In practice this turns out to work very well. After we computed a spectral value to high enough order of precision it remains to check that the third trace $t_{M_{1} M_{3}}$ is also real.

In the following we carry out the computations described above for a number of values of $g_{2}, g_{3}, m, n$ and compare them with the asymptotic approximations given by (9).

First we take $g_{2}=4, g_{3}=0$. This corresponds to the elliptic curve $y^{2}=4 x^{3}-4 x$ which has a square lattice. In this case equation (1) has an extra symmetry with respect to $x \rightarrow-x, B \rightarrow$ $-B$. Hence the spectrum of ( 7$)$ has the symmetry $B \rightarrow-B$. An obvious spectral value is $B=$ 0 . In that case we can write down an explicit solution for (1), namely ${ }_{2} F_{1}\left(1 / 8,1 / 8,3 / 4 ; z^{2}\right)$. It is well-known that second order differential equations for hypergeometric functions have triangle groups as monodromy groups, and triangle groups are unitary.

The periods read

$$
\omega_{1}=2 \int_{-1}^{0} \frac{d x}{2 \sqrt{x^{3}-x}}=2.62206 \cdots
$$

and $\omega_{2}=i \omega_{1}=2.62206 i$. The quasi-periods read

$$
\eta_{1}=-2 \int_{-1}^{0} \frac{x d x}{2 \sqrt{x^{3}-x}}=1.19814 \cdots
$$

and $\eta_{2}=-i \eta_{1}=-1.19814 i$. We check that $\eta_{1} \overline{\omega_{2}}-\eta_{2} \overline{\omega_{1}}=0$ and $\pi / 4 \Delta=0.114237$. Hence (9) yields the approximated eigenvalues

$$
1.43554(m+n i)^{2}+0.114237 \frac{m+n i}{m-n i} .
$$

Here is a table with some asymptotic and numerical eigenvalues for the eigenvalue problem (7) with $g_{2}=4, g_{3}=0$. In addition we list the traces of $A=M_{1} M_{2}, B=M_{1} M_{3}, C=M_{2} M_{3}$. 


\begin{tabular}{|c|c|l|l|c|c|c|}
\hline$m$ & $n$ & $\begin{array}{l}\text { numerical } \\
\text { value }\end{array}$ & $\begin{array}{l}\text { asymptotic } \\
\text { value }\end{array}$ & $t_{A}$ & $t_{B}$ & $t_{C}$ \\
\hline 1 & 0 & 1.5526 & 1.5497 & -2.0046 & 29.5424 & -29.6103 \\
2 & 0 & 5.8568 & 5.8564 & 2.00001 & 606.12 & 606.123 \\
1 & 1 & $2.9823 \mathrm{i}$ & $2.9853 \mathrm{i}$ & -26.191 & -26.191 & 683.914 \\
3 & 0 & 13.0343 & 13.0341 & -2 & 13463.8 & -13463.8 \\
2 & 1 & $4.3752+5.8326 \mathrm{i}$ & $4.3751+5.8335 \mathrm{i}$ & 24.3597 & -591.325 & -14380.1 \\
1 & 2 & $-4.3752+5.8326 \mathrm{i}$ & $-4.3751+5.8335 \mathrm{i}$ & -591.325 & 24.3597 & -14380.1 \\
3 & 1 & $11.5758+8.6814 \mathrm{i}$ & $11.5757+8.6817 \mathrm{i}$ & -23.7666 & -13352.8 & 316787 \\
2 & 2 & $11.5978 \mathrm{i}$ & $11.5986 \mathrm{i}$ & 569.847 & 569.847 & 324724 \\
1 & 3 & $-11.5758+8.6814 \mathrm{i}$ & $-11.5757+8.6817 \mathrm{i}$ & -13352.8 & -23.7665 & 316787 \\
3 & 2 & $7.22167+17.3315 \mathrm{i}$ & $7.22164+17.3319 \mathrm{i}$ & -556.427 & 13125.2 & -7303200 \\
2 & 3 & $-7.22167+17.3315 \mathrm{i}$ & $-7.22164+17.3319 \mathrm{i}$ & 13125.2 & -556.426 & -7303200 \\
3 & 3 & $25.9536 \mathrm{i}$ & $25.954 \mathrm{i}$ & -12917.7 & -12917.7 & 166867000 \\
\hline
\end{tabular}

\section{The case $n=1$}

In the previous section we have considered the parameter choice $n=-1 / 2$ in our Lamé equation. We shall now concentrate on the case $n=1$. In that case we can write down explicit solutions and thus provide evidence for the correctness of the asymptotic analysis we carried out for general $n$ and in particular $n=-1 / 2$.

So we consider the equation

$$
\frac{d^{2} y}{d z^{2}}-(2 \wp(z)+B) y=0 .
$$

Choose $a$ such that $B=\wp(a)$ and let us suppose that $2 a$ is not in the period lattice. In that case two independent solutions of the Lamé equation are given by $f(z), f(-z)$ where

$$
f(z)=e^{\zeta(a) z} \frac{\sigma(z-a)}{\sigma(z)}
$$

and $\sigma(z)$ is the Weierstrass $\sigma$-function defined by

$$
\sigma(z)=z \prod_{\omega \in \Lambda}^{\prime}\left(1-\frac{z}{\omega}\right) \exp \left(\frac{z}{\omega}+\frac{z^{2}}{2 \omega^{2}}\right) .
$$

It is a holomorphic function on $\mathbb{C}$ with zeros in the lattice points of $\Lambda$. Furthermore, for any $\omega \in \Lambda$,

$$
\sigma(z+\omega)= \pm e^{\eta(\omega)(z+\omega / 2)} \sigma(z)
$$

where we use + if $\omega / 2 \in \Lambda$ and - if not. It follows from this functional equation that

$$
f(z+\omega)=e^{\zeta(a) \omega-a \eta} f(z)
$$

Notice by the way that $f(z)$ is independent of the choice of $a$ modulo $\Lambda$. We now study for which values of $a$, and hence $B$, the Lamé equation allows a non-trivial real valued $\Lambda$-periodic solution, symmetric in $z$. 
A basis for the real valued symmetric solutions is given by

$$
U(z)=f(z) \overline{f(-z)}+f(-z) \overline{f(z)}, \quad V(z)=|f(z)|^{2}+|f(-z)|^{2}
$$

Suppose that $f(z+\omega)=\lambda f(z)$. Then $f(-z-\omega)=\lambda^{-1} f(-z)$. The $\Lambda$-periodic linear combinations of $U(z), V(z)$ are either $U(z)$ itself if $\lambda=\bar{\lambda}$ for all $\omega \in \Lambda$ or $V(z)$ if $|\lambda|=1$ for all $\omega$. Suppose $f\left(z+\omega_{j}\right)=\lambda_{j} f(z)$ for $j=1,2$. Then the first case corresponds to $\lambda_{1}, \lambda_{2} \in \mathbb{R}$ and the second to $\left|\lambda_{1}\right|=\left|\lambda_{2}\right|=1$.

Let us consider the second case first. We have necessarily

$$
\zeta(a) \omega_{1}-\eta_{1} a=-2 \pi i r_{2}, \quad \zeta(a) \omega_{2}-\eta_{2} a=2 \pi i r_{1}
$$

with $r_{1}, r_{2} \in \mathbb{R}$. Solving this for $a$ and $\zeta(a)$ we get

$$
\zeta(a)=r_{1} \eta_{1}+r_{2} \eta_{2}, \quad a=r_{1} \omega_{1}+r_{2} \omega_{2} .
$$

Hence we must solve

$$
\zeta\left(r_{1} \omega_{1}+r_{2} \omega_{2}\right)=r_{1} \eta_{1}+r_{2} \eta_{2}
$$

in $r_{1}, r_{2} \in \mathbb{R}$. Notice that $\zeta\left(x_{1} \omega_{1}+x_{2} \omega_{2}\right)-x_{1} \eta_{1}-x_{2} \eta_{2}$ is periodic in $x_{1}, x_{2}$ with period 1 , so we can restrict ourselves to finding solutions with $0 \leq r_{1,2}<1$. We expect at most finitely many solutions.

Let us now consider the case when $\lambda_{1}, \lambda_{2} \in \mathbb{R}$. This implies that there exist integers $m_{1}, m_{2}$ such that

$$
\begin{aligned}
& \zeta(a) \omega_{1}-\overline{\zeta(a) \omega_{1}}-a \eta_{1}+\overline{a \eta_{1}}=-2 \pi m_{2} \\
& \zeta(a) \omega_{2}-\overline{\zeta(a) \omega_{2}}-a \eta_{2}+\overline{a \eta_{2}}=2 \pi m_{1}
\end{aligned}
$$

We solve this equation recursively in $a$ for large values of $\left|m_{1}\right|+\left|m_{2}\right|$. The observation is that $a$ should be close to a lattice point, which we can take to be 0 . So let us put $l=\zeta(a)$ and notice that $a=1 / l+O\left(1 /|l|^{2}\right)$. So our equation can be rewritten as

$$
\begin{aligned}
& l \omega_{1}-\overline{l \omega_{1}}-\frac{\eta_{1}}{l}+\frac{\overline{\eta_{1}}}{\bar{l}}=-2 \pi i m_{2}+O\left(|l|^{-2}\right) \\
& l \omega_{2}-\overline{l \omega_{2}}-\frac{\eta_{2}}{l}+\frac{\overline{\eta_{2}}}{\bar{l}}=2 \pi i m_{1}+O\left(|l|^{-2}\right)
\end{aligned}
$$

Notice that this precisely the problem (10) for $n=1$. The difference is now that we have convergence instead of asymptotic approximation. Our conclusion follows by noticing that $B=\wp(a)=\zeta(a)^{2}+O(1 /|\zeta(a)|)=l^{2}+O(1 /|l|)$ for $a$ very close to 0 . So we have,

Theorem 7.1 Conjecture 5.1 is true when $n=1$.

\section{References}

[Be2002 ] Beukers, On Dwork's accessory parameter problem, Math. Z. 241(2002), 425-444.

[Hi1908 ] E.Hilb, Über Kleinsche Theoreme in der Theorie der linearen Differentialgleichungen, Math.Ann. 66(1909), 215-257. 
[K11907 ] F. Klein, Bemerkungen zur Theorie der linearen Differentialgleichungen zweiter Ordnung, Math. Ann. 64 (1907), 175-196.

[KRV1979 ] L.Keen, H.E.Rauch, A.T.Vasquez, Moduli of punctured tori and the accessory parameter of Lamé's equation, Trans.AMS 255(1979), 201-230.

[MSW2002 ] D.Mumford, C.Series, D.Wright, Indra's Pearls, The vision of Felix Klein, Cambridge University Press 2002.

[Ne1949 ] Z. Nehari, On the accessory problem of a Fuchsian differential equation, Amer.J.Math. 71(1949), 24-39.

[WW1980 ] E.T.Whittaker, G.N.Watson, A course on Modern Analysis, 4th edition, Cambridge University Press, 1980. 